\title{
Association of risk factors and bleeding complications in Asian patients taking edoxaban
}

\author{
Ok Sang Lee ${ }^{1}$, Woorim Kim${ }^{1}$, Bo Min Jang ${ }^{2}$, Kyung Hyun Min ${ }^{1}$, Yoon Sook Cho ${ }^{2}$, Myung \\ Koo Lee ${ }^{1}$, Sandy Rhie ${ }^{3}$, and Kyung Eun Lee ${ }^{1}$ \\ ${ }^{1}$ Chungbuk National University \\ ${ }^{2}$ Seoul National University Hospital \\ ${ }^{3}$ Ewha Womans University
}

July 7, 2020

\begin{abstract}
Aim: Asian patients are known to be more prone to bleeding complications than patients of other ethnicities. Therefore, there are possibilities of other risk factors that should be given special consideration for dosage adjustment in this specific ethnic group. This study aimed to investigate the risk factors for bleeding complications in Asian patients under appropriate edoxaban dosage regimens. Methods: Data on patients taking proper dosages, based on the Lixiana package insert, were analyzed. Univariate and multivariable analyses were conducted to evaluate associations between risk factors and bleeding outcomes. Subgroup analysis was performed on high-risk patients for bleeding complications whose edoxaban dose was reduced according to the package insert. Results: A total of 346 patients were included. Among them, 32 patients experienced bleeding complications. Patients with either weights of less than or equal to $60 \mathrm{~kg}$ and with cancer showed around 3.3- and 3.4-fold increased risk of bleeding complications compared to heavier patients ( $>60 \mathrm{~kg}$ ) and those without cancer, respectively. In subgroup analysis with high-risk patients who took low-dose edoxaban (15 mg and $30 \mathrm{mg}$ ), weights of less than or equal to 60 $\mathrm{kg}$ remained a significant factor for bleeding outcomes. Conclusion: This study showed that weights of less than or equal to 60 $\mathrm{kg}$ and the presence of cancers could affect bleeding complications which occurred despite proper edoxaban treatment in Asian patients. Therefore, more strict dosage guideline could be considered in populations with high proportions of Asian ethnicities.
\end{abstract}

\section{Introduction}

Direct oral anticoagulants (DOACs) are widely used for treatment and prophylaxis of atrial fibrillation $(\mathrm{AF})$, ischemic stroke, deep vein thrombosis (DVT), and pulmonary embolism (PE). The recent 2019 AHA/ACC/HRS Focused Update of the 2014 guidelines for management of patients with AF states that DOACs are recommended over warfarin in eligible patients [1]. Although warfarin has been the most widely used oral anticoagulant, due to its shortcomings including a narrow therapeutic range and wide inter-and intra-individual variability [2], DOACs are currently preferred in many clinical settings [3, 4].

Among DOACs, edoxaban is the most recently introduced drug [5] and has mainly been used as an alternative to other DOACs. However, after many studies showed that both high-dose and low-dose edoxaban were not inferior to warfarin for stroke prevention and associated with a significantly lower incidence of bleeding complications [6-9], edoxaban became a representative DOAC [1]. Hence, edoxaban is likely to be prescribed more widely in the future, depending on the clinical setting or patient profile.

Bleeding complication is one of the major concerns during edoxaban usage [10]. The generally recommended dose of edoxaban for treatment of DVT/PE and AF is $60 \mathrm{mg}$ once daily. In order to reduce the incidence of bleeding complications, patients with creatinine clearance $(\mathrm{CrCl}) 15$ to $50 \mathrm{~mL} / \mathrm{min}$, weights of less than or equal to $60 \mathrm{~kg}$, or who are taking certain concomitant P-glycoprotein (P-gp) inhibitors are recommended 
to take $30 \mathrm{mg}$ once daily for treatment of DVT and PE instead [5]. However, Asian patients are known to be more prone to bleeding complications than patients of other ethnicities [11], opening possibilities of other risk factors that should be given special consideration for dosage adjustment in this specific ethnic group. In this context, this study aimed to provide evidence of the association between risk factors and bleeding complications in Asian patients with an appropriate edoxaban dosage regimen.

\section{Methods}

\section{Study patients and data collection}

This study consists of 510 patients who were prescribed by Seoul National University Hospital with edoxaban between March 1, 2016 and June 30, 2017. Patients who were 18 or older and received proper dosage of edoxaban based on Lixiana package insert (Korean version) [12] were eligible for the study. Patients who received inappropriate dose of edoxaban were excluded. Data collection was conducted using electronic medical records. Data on age, sex, weight, serum creatinine, comorbidities, concurrent medication, international normalized ratio (INR) measurements, history of bleeding complications, and liver function test (cirrhosis or bilirubin $>2 \mathrm{x}$ normal, or aspartate aminotransferase/alanine aminotransferase/alkaline phosphatase $>3 \mathrm{x}$ normal) were collected. Bleeding complications were classified as major, clinically relevant non-major, or minor bleeding using the scheme detailed in the International Society on Thrombosis and Hemostasis criteria [13].

This study was approved by the Institutional Review Board of the Seoul National University Hospital (approval number: H-1809-044-971).

\section{Statistical analysis}

Chi-square test or Fisher's exact test was used to compare categorical variables between patients with bleeding complications and those without complications. Multivariable logistic regression analysis was used to examine independent risk factors for bleeding complications. Factors having a p-value less than 0.05 in univariate analysis along with clinically relevant confounders (age, sex, and $\mathrm{CrCl}$ ) were included in multivariable analysis. Subgroup analysis was conducted on high-risk patients for bleeding complications whose edoxaban dose was reduced to less than or equal to $30 \mathrm{mg}$. Odds ratios and adjusted odds ratios were calculated through univariate and multivariable analyses, respectively. A p-value of less than 0.05 was considered statistically significant. To test the model's goodness of fit, we performed a Hosmer-Lemeshow test. All statistical analyses were conducted using IBM SPSS statistics, version 20 software (International Business Machines Corp., New York, USA).

\section{Results}

Among the 510 patient enrolled in this study, 164 patients were excluded due to the usage of inappropriate dose of edoxaban. Accordingly, data on 346 patients, who were receiving recommended dosages of edoxaban, were used for the analysis. The median age of the included patients was 73 years (range, 34-93 years), and there were 168 (48.6\%) females. Thirty two patients (9.2\%) experienced bleeding complications after taking edoxaban. Among them, 16 patients experienced major bleeding complications. Two and eight patients experienced intracranial hemorrhage and gastrointestinal bleeding complications, respectively. Six patients showed urological bleeding and 11 had unclassified bleeding outcomes. Eleven patients experienced stroke or recurrent DVT/PE.

As shown in Table 1, patients less than or equal to $60 \mathrm{~kg}$ had more bleeding complications than those who were heavier than $60 \mathrm{~kg}(\mathrm{p}=0.018)$. Also, patients with cancer had more bleeding outcomes than those without cancer $(\mathrm{p}=0.030)$.

Multivariable analysis (Table 2) included sex, age, $\mathrm{CrCl}$, and factors with $\mathrm{p}<0.05$ in univariate analysis (weight and cancer). After adjusting for related covariates, patients with cancer or weights less than or equal to $60 \mathrm{~kg}$ showed approximately 3.4-fold higher bleeding complications than patients without cancer or 
with weights higher than $60 \mathrm{~kg}$. The Hosmer-Lemeshow test showed that the fitness of the multivariable analysis model was satisfactory $\left(\chi^{2}=11.335,6\right.$ degrees of freedom, $\left.\mathrm{p}=0.079\right)$.

Since more than $10 \%$ of patients had bleeding complications even after dose reduction, we performed subgroup analysis to investigate factors on bleeding complications in high-risk patients receiving a low dose of edoxaban. We found that weight was a significant factor for bleeding outcomes in both univariate and multivariable analysis (Table 3 and Table 4). Patients with weight [?]60 kg showed 3.3-times higher bleeding complications even with low dose of edoxaban compared to those with weight $>60 \mathrm{~kg}$. Hosmer-Lemeshow test showed that the fitness of the multivariable analysis model was satisfactory $\left(\chi^{2}=3.636,6\right.$ degrees of freedom, $\left.\mathrm{p}=0.726\right)$.

\section{Discussion}

The main finding of this study is that weight ([?] $60 \mathrm{~kg}$ ) and cancer were associated with bleeding complications under appropriate edoxaban dosage regimen. Patients with weights of less than or equal to $60 \mathrm{~kg}$ and cancer had around a 3.4-fold increased risk of bleeding complications compared to heavier patients $(>60$ $\mathrm{kg}$ ) and those without cancer. In the subgroup analysis with high-risk patients who took low-dose edoxaban $(15 \mathrm{mg}$ and $30 \mathrm{mg}$ ), weights of less than or equal to $60 \mathrm{~kg}$ was remained a significant factor for bleeding outcomes than those who weigh more than $60 \mathrm{~kg}$.

Edoxaban is implicated in the reduction of stroke and systemic embolism risks in nonvalvular AF and treatment of DVT and PE [5]. According to the 2019 AHA/ACC/HRS Guideline for the Management of $\mathrm{AF}$, edoxaban has been added to the list of DOACs used for stroke prevention and is recommended over warfarin except in patients with moderate to severe mitral stenosis or prosthetic heart valves [1]. Several studies have shown that edoxaban was not inferior to warfarin regarding the prevention of stroke or systemic embolization and was associated with significantly lower rates of bleeding and death from cardiovascular causes [6-9]. Yet, although edoxaban is the preferred oral anticoagulant over warfarin, it still can increase the risk of bleeding and can cause serious or even potentially fatal bleeding. Hence, risk factors should be carefully considered when administering edoxaban.

It has been reported that Asians exhibit more bleeding risks than other ethnicities; a study showed that death from hemorrhagic stroke was more common in Asian patients compared to others [14]. Reports of bleeding complications with antithrombotic agents also consistently revealed that Asians experienced more bleeding complications than other ethnic groups. A meta-analysis showed that DOAC-associated intracranial hemorrhage (ICH) was significantly higher in the Asian population [15]. In addition, in the case of warfarin treatment, a retrospective cohort study using AF patients showed that the incidence for ICH in Asians was 4.06-times higher than in Caucasians [16]. For antiplatelet therapy, a meta-analysis showed that bleeding events occurred twice as frequently in Asians than in the non-Asian group [17].

Most dosage studies of oral anticoagulants have primarily involved Caucasians; therefore, current edoxaban dosage regimens may have been predominantly derived from data on white patients. This study showed that standard dosage regimens of edoxaban induced complications in Asian individuals, which may stem from ethnicity-dependent profiles. The U.S. Savaysa package insert suggested that DVT/PE patients with $\mathrm{CrCl} 15$ to $50 \mathrm{~mL} / \mathrm{min}$, weights of less than or equal to $60 \mathrm{~kg}$, or who are taking certain concomitant P-gp inhibitors are recommended to take $30 \mathrm{mg}$ once daily [5]. Also, for patients with nonvalvular AF, $\mathrm{CrCl} 15-50$ $\mathrm{mL} / \mathrm{min}$ was the sole criterion for dose reduction [5]. In contrast, the Korean package insert suggests that patients with $\mathrm{AF}$, in addition to DVT/PE, should reduce edoxaban dose under two more criteria, namely lower weight ([?] $60 \mathrm{~kg}$ ) and $\mathrm{P}$-gp inhibitor concomitant usage, alongside $\mathrm{CrCl} 15-50 \mathrm{~mL} / \mathrm{min}$. [12]. Thus it may be stated that considering high bleeding risks of Asian populations, the Korean dose recommendation is much more conservative.

In this study, subgroup analyses showed that 27 patients still experienced bleeding complications despite dosage adjustments. It has been previously established that the incidence of bleeding events during edoxaban administration shows a dose-dependent trend [18]. Moreover, Yamashita et al. reported body weightdependent differences in the incidence of bleeding risks, suggesting that weight is an important factor to consider in assessing the risk of bleeding while taking edoxaban [18]. Hence, for ethnic groups that are known 
to be more prone to bleeding complications, modification of anticoagulation intensity may be considered. Since the current results showed that lower weight was a significant risk factor for bleeding outcome even in patients using low-dose edoxaban, more strict dosage guidelines for patients with lower weights could be beneficial or perhaps necessary.

This study revealed that cancer was significantly associated with bleeding complications for patients with edoxaban therapy. Cancer patients with anticoagulation are more prone to bleeding outcomes compared to those without cancer. A study showed that cancer patients on anticoagulation therapy had approximately 2.2-fold higher bleeding complications than those without malignancies [19]. Another retrospective analysis concluded that the incidence of bleeding complications for cancer patients was higher than in patients without cancer. Hutten et al. showed that patients with cancer, compared with nonmalignant patients, had a statistically significantly increased overall incidence (100 patient-years) of recurrence (27.1 vs 9.0, respectively) as well as bleeding (13.3 vs 2.1, respectively) [20]. Our study was also consistent with the findings that patients with cancer showed a 3.4 -fold increased risk of bleeding complications than those without cancer.

It is possible that bleeding is caused by the cancer itself, as tumor invasion, abnormal tumor vasculature or tumor regression can increase risk of bleeding [21]. Furthermore, anti-tumor treatment including chemotherapy and anti-inflammatory drugs can exacerbate bleeding outcomes. Finally, cancer patients with thrombocytopenia from malignancies or chemotherapy may result in bleeding complications [21]. In this context, cancer should be considered for dosage adjustment when administering edoxaban. Moreover, more frequent monitoring in this high-risk group may be recommended.

The limitations of our study are that it was conducted in a single center and designed retrospectively. Another shortcoming is a lack of information on detailed mechanisms and genetic polymorphisms. Nevertheless, this study revealed potential risk factors for bleeding complications in Asian patients undergoing edoxaban therapy. In addition, this study provided preliminary data to modify current dosage regimen to reduce the risk of bleeding outcomes; these factors can be applied for developing individualized drug therapy with edoxaban.

\section{Authorship}

Conceptualization: Ok Sang Lee, Woorim Kim, Bo Min Jang, Sandy Jeong Rhie, Kyung Eun Lee; Methodology: Ok Sang Lee, Woorim Kim; Formal analysis and investigation: Ok Sang Lee, Woorim Kim, Kyung Hyun Min ; Writing - original draft preparation: Woorim Kim, Ok Sang Lee; Writing - review and editing: Myung Koo Lee, Kyung Eun Lee; Funding acquisition: Kyung Eun Lee; Resources: Bo Min Jang, Yoon Sook Cho; Supervision: Kyung Eun Lee

All authors have read and approved the final version of this manuscript.

\section{Conflict of interest statement}

The authors declare that they have no conflict of interest.

\section{Funding information}

This work was supported by the Medical Research Center Program (2017R1A5A2015541) of the NRF funded by the Korean government (MSIP). The funding sources did not have a role in the design, conduct, and analysis of the study.

\section{Reference}

[1] January CT, Wann LS, Calkins H, et al. 2019 Focused update on atrial fibrillation AHA/ACC/HRS focused update of the $2014 \mathrm{AHA} / \mathrm{ACC} / \mathrm{HRS}$ guideline for the management of patients with atrial fibrillation: a report of the American college of cardiology/American heart association task force on clinical practice guidelines and the heart rhythm society. Circulation 2019; 139:e125-51. 
[2] Ansell J, Hirsh J, Hylek E, et al. American college of chest physicians. Pharmacology and management of the vitamin $\mathrm{K}$ antagonists: American college of chest physicians evidence-based clinical practice guidelines (8th Edition). Chest 2008; 133:160S-98S.

[3] Boom MS, Berghuis EM, Nieuwkerk PT, et al. When do patients prefer a direct oral anticoagulant over a vitamin K antagonist? Neth J Med 2015; 73:368-72.

[4] Brekelmans MPA, Kappelhof M, Nieuwkerk PT, et al. Preference for direct oral anticoagulants in patients treated with vitamin K antagonists for venous thromboembolism. Neth J Med 2017; 75:50-5.

[5] SAVAYSA@ [package insert], Basking Ridge, NJ: Daiichi Sankyo, Inc.; 2020.

[6] Giugliano RP 1, Ruff CT, Braunwald E, et al. Edoxaban versus warfarin in patients with atrial fibrillation. N Engl J Med 2013; 369:2093-104.

[7] Fiedler KA, Maeng M, Mehilli J, et al. Duration of triple Therapy in patients requiring oral anticoagulation after drug-eluting stent implantation: the ISAR-TRIPLE trial. J Am Coll Cardiol 2015; 65:1619-29.

[8] Hariharan S, Madabushi R. Clinical pharmacology basis of deriving dosing recommendations for dabigatran in patients with severe renal impairment. J Clin Pharmacol 2012; 52:119S-25S.

[9] Mikkelsen AP, Lindhardsen J, Lip GYH, et al. Female sex as a risk factor for stroke in atrial fibrillation: a nationwide cohort study. J Thromb Haemost 2012; 10:1745-51.

[10] Stacy ZA, Call WB, Hartmann AP, Peters GL, Richter SK. Edoxaban: a comprehensive review of the pharmacology and clinical data for the management of atrial fibrillation and venous thromboembolism. Cardiol Ther 2016; 5:1-18.

[11] Oh YB, Hong KS, Heo JH et al. New oral anticoagulants may be particularly useful for Asian stroke patients. J Stroke 2014; 16:73-80.

[12] LIXIANA@ [package insert], Seoul, Korea: Daiichi Sankyo Korea Co., Ltd.; 2020.

[13] Schulman S, Kearon C. Subcommittee on control of anticoagulation of the scientific and standardization committee of the international society on thrombosis and haemostasis. definition of major bleeding in clinical investigations of antihemostatic medicinal products in non-surgical patients. J. Thromb Haemost 2005; $3: 3692-4$.

[14] Ayala C, Croft JB, Greenlund KJ, et al. Sex differences in US mortality rates for stroke and stroke subtypes by race/ethnicity and age, 1995-1998. Stroke 2002; 33:1197-201.

[15] Wang KL, Lip GY, Lin SJ, Chiang CE. Non-Vitamin K antagonist oral anticoagulants for stroke prevention in Asian patients with nonvalvular atrial fibrillation: meta-analysis. Stroke 2015; 46:2555-61.

[16] Shen AY, Yao JF, Brar SS, Jorgensen MB, Chen W. Racial/ethnic differences in the risk of intracranial hemorrhage among patients with atrial fibrillation. J Am Coll Cardiol 2007; 50:309-15.

[17] Kang J, Park KW, Palmerini T, et al. Racial differences in ischaemia/bleeding risk trade-off during anti-platelet therapy: individual patient level landmark meta-analysis from seven RCTs. Thromb Haemost $2019 ; 119: 49-162$.

[18] Yamashita T, Koretsune Y, Yasaka M, et al. Randomized, multicenter, warfarin-controlled phase II study of edoxaban in Japanese patients with non-valvular atrial fibrillation. Circ J 2012; 76:1840-7.

[19] Prandoni P, Lensing AWA, Piccioli A, et al. Recurrent venous thromboembolism and bleeding complications during anticoagulant treatment in patients with cancer and venous thrombosis. Blood 2002; 100:3484-8.

[20] Hutten BA, Prins MH, Gent M, Ginsberg J, Tijssen JG, Büller HR. Incidence of recurrent thromboembolic and bleeding complications among patients with venous thromboembolism in relation to both malignancy and achieved international normalized ratio: a retrospective analysis. J Clin Oncol 2000; 18:3078-83. 
[21] Johnstone C, Rich SE. Bleeding in cancer patients and its treatment: a review. Ann Palliat Med 2018; 7:265-73.

Table 1. Factors associated with bleeding events caused by edoxaban use

\begin{tabular}{|c|c|c|c|}
\hline & No events $\mathrm{N}=314$ & Events $\mathrm{N}=32$ & $p$-value \\
\hline \multicolumn{4}{|c|}{ Age (years) } \\
\hline$<65$ & $69(22.0)$ & $3(9.4)$ & 0.094 \\
\hline [?] 65 & $245(78.0)$ & $29(90.6)$ & \\
\hline \multicolumn{4}{|l|}{ Sex } \\
\hline Male & $165(52.5)$ & $13(40.6)$ & 0.199 \\
\hline Female & $149(47.5)$ & $19(59.4)$ & \\
\hline \multicolumn{4}{|c|}{ Weight (kg) } \\
\hline [?] 60 & $119(37.9)$ & $19(59.4)$ & 0.018 \\
\hline$>60$ & $195(62.1)$ & $13(40.6)$ & \\
\hline \multicolumn{4}{|c|}{$\mathrm{CrCl}(\mathrm{mL} / \mathrm{min})$} \\
\hline $15-50$ & $91(34.5)$ & $8(29.6)$ & 0.613 \\
\hline$>50$ & $173(65.5)$ & $19(70.4)$ & \\
\hline \multicolumn{4}{|c|}{ Medical history } \\
\hline \multicolumn{4}{|c|}{$\begin{array}{l}\text { Congestive heart } \\
\text { failure }\end{array}$} \\
\hline Yes & $49(15.6)$ & $2(6.2)$ & 0.196 \\
\hline No & $265(84.4)$ & $30(93.8)$ & \\
\hline \multicolumn{4}{|c|}{ Hypertension } \\
\hline Yes & $204(65.0)$ & $16(50.0)$ & 0.094 \\
\hline No & $110(35.0)$ & $16(50.0)$ & \\
\hline \multicolumn{4}{|c|}{ Diabetes } \\
\hline Yes & $101(32.2)$ & $9(28.1)$ & 0.640 \\
\hline No & $213(67.8)$ & $23(71.9)$ & \\
\hline \multicolumn{4}{|c|}{$\begin{array}{l}\text { Previous ischemic } \\
\text { stroke or TIA }\end{array}$} \\
\hline Yes & $47(15.0)$ & $5(15.6)$ & 1.000 \\
\hline No & $267(85.0)$ & $27(84.4)$ & \\
\hline \multicolumn{4}{|c|}{ Vascular disease } \\
\hline Yes & $4(1.3)$ & $1(3.1)$ & 0.386 \\
\hline No & $310(98.7)$ & $31(96.9)$ & \\
\hline \multicolumn{4}{|c|}{ Immobilization } \\
\hline Yes & $7(2.2)$ & $1(3.1)$ & 0.544 \\
\hline No & $307(97.8)$ & $31(96.9)$ & \\
\hline \multicolumn{4}{|c|}{$\begin{array}{l}\text { Recent ([?]1 month) } \\
\text { trauma and/or surgery }\end{array}$} \\
\hline Yes & $4(1.3)$ & $0(0.0)$ & 1.000 \\
\hline No & $310(98.7)$ & $32(100.0)$ & \\
\hline \multicolumn{4}{|c|}{$\begin{array}{l}\text { Abnormal liver } \\
\text { function }^{a}\end{array}$} \\
\hline Yes & $10(3.2)$ & $2(6.2)$ & 0.306 \\
\hline No & $304(96.8)$ & $30(93.8)$ & \\
\hline \multicolumn{4}{|l|}{ INR } \\
\hline Normal & $168(53.5)$ & $18(56.2)$ & 0.767 \\
\hline High $^{\mathrm{b}}$ & $146(46.5)$ & $14(43.8)$ & \\
\hline \multicolumn{4}{|c|}{ Previous bleeding } \\
\hline Yes & $43(13.7)$ & $8(25.0)$ & 0.112 \\
\hline
\end{tabular}




\begin{tabular}{llll}
\hline & No events N $=314$ & Events N $=32$ & $p$-value \\
\hline $\begin{array}{l}\text { No } \\
\text { Cancer }\end{array}$ & $271(86.3)$ & $24(75.0)$ & \\
Yes & $28(8.9)$ & $7(21.9)$ & 0.030 \\
No & $286(91.1)$ & $25(78.1)$ & \\
Medication & & & \\
Antiplatelet agents & $20(6.4)$ & $3(9.4)$ & 0.459 \\
Yes & $294(93.6)$ & $29(90.6)$ & \\
No & $7(2.2)$ & $1(3.1)$ & 0.544 \\
Steroids & $307(97.8)$ & $31(96.9)$ & \\
Yes & & & \\
No & &
\end{tabular}

CrCl: Creatinine clearance; TIA: Transient ischemic attack; INR: International normalized ratio

${ }^{a}$ Cirrhosis or bilirubin $>2 \times$ normal, or aspartate aminotransferase/alanine aminotransferase/alkaline phosphatase $>3 \times$ normal

${ }^{\mathrm{b}} \mathrm{INR}>1.1$

Table 2. Multivariable analysis to identify predictors of bleeding complications

\begin{tabular}{lllll}
\hline & Crude OR $(95 \% \mathrm{CI})$ & $p$-value & Adjusted $^{*}$ OR $(95 \% \mathrm{CI})$ & $p$-value \\
\hline Female & $1.618(0.773-3.390)$ & 0.202 & & \\
Age [?] 65 & $2.722(0.805-9.206)$ & 0.107 & & \\
CrCl 15-50 (mL/min) & $0.800(0.337-1.900)$ & 0.614 & & \\
Weight [?] 60 kg & $2.395(1.141-5.027)$ & 0.021 & $3.378(1.372-8.312)$ & 0.008 \\
Cancer & $2.860(1.136-7.203)$ & 0.026 & $3.429(1.275-9.224)$ & 0.015 \\
\hline
\end{tabular}

$\mathrm{CrCl}$ : Creatinine clearance

*Adjusted for sex, age, $\mathrm{CrCl}$, weight, and cancer

Table 3. Subgroup analysis of factors associated with bleeding events in patients on low-dose edoxaban

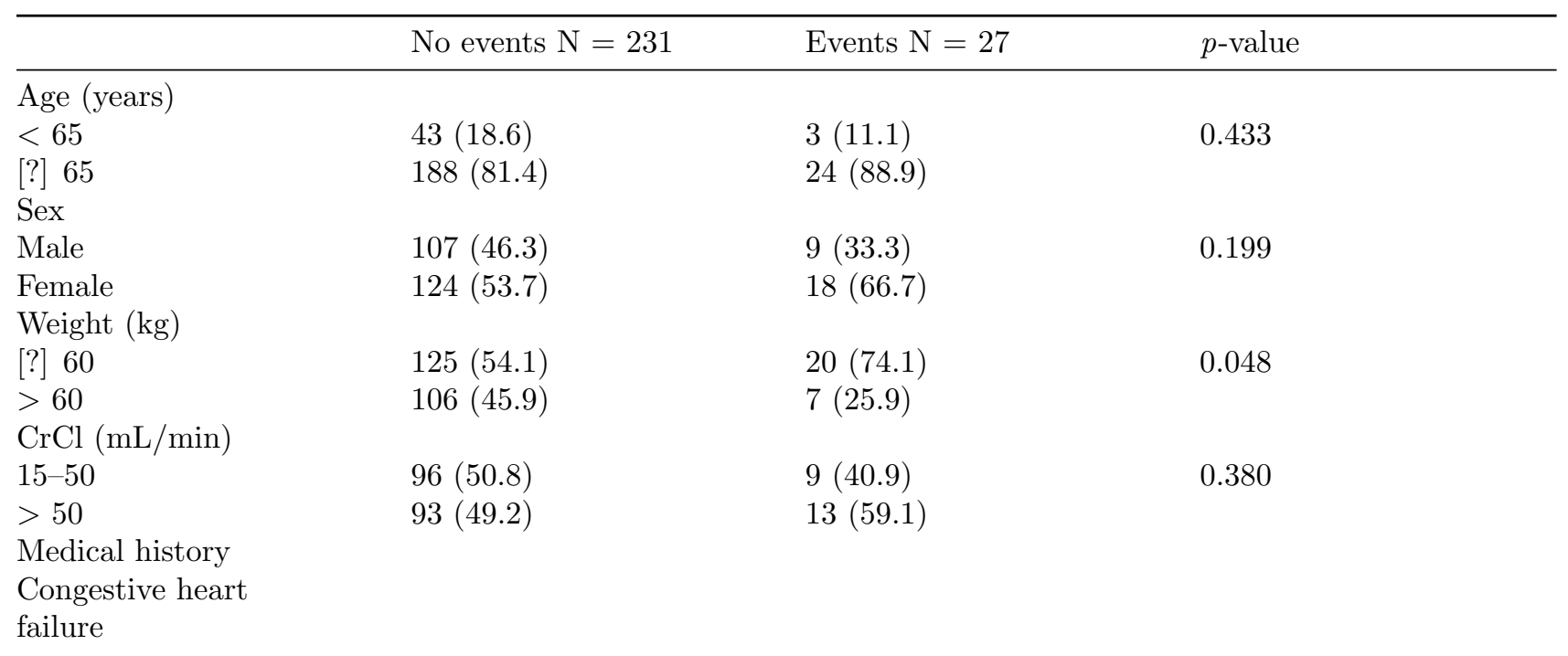




\begin{tabular}{|c|c|c|c|}
\hline & No events $\mathrm{N}=231$ & Events $\mathrm{N}=27$ & $p$-value \\
\hline Yes & $36(15.9)$ & $3(11.1)$ & 0.777 \\
\hline No & $191(84.1)$ & $24(88.9)$ & \\
\hline \multicolumn{4}{|c|}{ Hypertension } \\
\hline Yes & $139(61.2)$ & $14(51.9)$ & 0.346 \\
\hline No & $88(38.8)$ & $13(48.1)$ & \\
\hline \multicolumn{4}{|c|}{ Diabetes } \\
\hline Yes & $66(29.1)$ & $7(25.9)$ & 0.732 \\
\hline No & $161(70.9)$ & $20(74.1)$ & \\
\hline \multicolumn{4}{|c|}{$\begin{array}{l}\text { Previous ischemic } \\
\text { stroke or TIA }\end{array}$} \\
\hline Yes & $44(19.4)$ & $6(22.2)$ & 0.726 \\
\hline No & $183(80.6)$ & $21(77.8)$ & \\
\hline \multicolumn{4}{|c|}{ Vascular disease } \\
\hline Yes & $2(0.9)$ & $1(3.7)$ & 0.283 \\
\hline No & $229(99.1)$ & $26(96.3)$ & \\
\hline \multicolumn{4}{|c|}{ Immobilization } \\
\hline Yes & $6(2.6)$ & $1(3.7)$ & 0.549 \\
\hline No & $221(97.4)$ & $26(96.3)$ & \\
\hline \multicolumn{4}{|c|}{$\begin{array}{l}\text { Recent ([?]1 month) } \\
\text { trauma and/or surgery }\end{array}$} \\
\hline Yes & $4(1.8)$ & $0(0.0)$ & 1.000 \\
\hline No & $223(98.2)$ & $27(100.0)$ & \\
\hline \multicolumn{4}{|c|}{ Abnormal liver } \\
\hline Yes & $4(1.7)$ & $1(3.7)$ & 0.427 \\
\hline No & $227(98.3)$ & $26(96.3)$ & \\
\hline \multicolumn{4}{|l|}{ INR } \\
\hline Normal & $127(55.0)$ & $116(59.3)$ & 0.672 \\
\hline $\operatorname{High}^{\mathrm{b}}$ & $104(45.0)$ & $11(40.7)$ & \\
\hline \multicolumn{4}{|c|}{ Previous bleeding } \\
\hline Yes & $35(15.4)$ & $7(25.9)$ & 0.174 \\
\hline No & $192(84.6)$ & $20(74.1)$ & \\
\hline \multicolumn{4}{|l|}{ Cancer } \\
\hline Yes & $20(8.7)$ & $5(18.5)$ & 0.157 \\
\hline No & $211(91.3)$ & $22(81.5)$ & \\
\hline \multicolumn{4}{|c|}{ Medication } \\
\hline \multicolumn{4}{|c|}{ Antiplatelet agents } \\
\hline Yes & $19(8.4)$ & $3(11.1)$ & 0.714 \\
\hline No & $208(91.6)$ & $24(88.9)$ & \\
\hline \multicolumn{4}{|l|}{ Steroids } \\
\hline Yes & $3(1.3)$ & $1(3.7)$ & 0.359 \\
\hline No & $228(98.7)$ & $26(96.3)$ & \\
\hline
\end{tabular}

CrCl: Creatinine clearance; TIA: Transient ischemic attack; INR: International normalized ratio

${ }^{\text {a }}$ Cirrhosis or bilirubin $>2 \times$ normal, or aspartate aminotransferase/alanine aminotransferase/alkaline phosphatase $>3 \times$ normal

${ }^{\mathrm{b}} \mathrm{INR}>1.1$

Table 4. Multivariable analysis of subgroup analysis of factors associated with bleeding events in patients on 
low-dose edoxaban

\begin{tabular}{lllll}
\hline & Crude OR $(95 \%$ CI $)$ & $p$-value & Adjusted $^{*}$ OR $(95 \% \mathrm{CI})$ & $p$-value \\
\hline Female & $1.726(0.744-4.001)$ & 0.203 & & \\
Age [?] 65 & $1.830(0.527-6.355)$ & 0.342 & & \\
CrCl $15-50 \mathrm{~mL} / \mathrm{min}$ & $0.671(0.274-1.645)$ & 0.382 & & \\
Weight [?] $60 \mathrm{~kg}$ & $2.395(1.141-5.027)$ & 0.021 & $3.265(1.078-9.886)$ & 0.036 \\
\hline
\end{tabular}

$\mathrm{CrCl}$ : Creatinine clearance

*adjusted for sex, age, $\mathrm{CrCl}$, and weight 